\title{
Communication
}

\section{Trifluoroethanol Promoted Castagnoli-Cushman Cycloadditions of Imines with Homophthalic Anhydride}

\author{
Thibault Bayles and Catherine Guillou *(D)
}

check for updates

Citation: Bayles, T.; Guillou, C. Trifluoroethanol Promoted Castagnoli-Cushman Cycloadditions of Imines with Homophthalic Anhydride. Molecules 2022, 27, 844. https://doi.org/10.3390/ molecules27030844

Received: 5 January 2022

Accepted: 25 January 2022

Published: 27 January 2022

Publisher's Note: MDPI stays neutral with regard to jurisdictional claims in published maps and institutional affiliations.

Copyright: (C) 2022 by the authors Licensee MDPI, Basel, Switzerland. This article is an open access article distributed under the terms and conditions of the Creative Commons Attribution (CC BY) license (https:// creativecommons.org/licenses/by/ $4.0 /)$.
Institut de Chimie des Substances Naturelles CNRS, Université de Paris-Saclay, 1 Avenue de la Terrasse, 91198 Gif-sur-Yvette, France; thibault.bayles@cnrs.fr

* Correspondence: catherine.guillou@cnrs.fr

\begin{abstract}
Lactams are essential compounds in medicinal chemistry and key intermediates in the synthesis of natural products. The Castagnoli-Cushman reaction (CCR) of homophthalic anhydride with imines is an exciting method for accessing cyclic densely substituted lactam products. Most CCRs need to be catalyzed or heated. Herein, we report a new, efficient, metal and catalyst-free CCR for the synthesis of poly-substituted 3,4-lactams utilizing the unique properties of trifluoroethanol (TFE). This procedure provides high-speed and smooth access to a broad range of densely substituted 3,4-lactams in good yields and a 100\% atom-economical fashion.
\end{abstract}

Keywords: Castagnoli-Cushman reaction; homophthalic anhydride; imines; lactam; trifluoroethanol

\section{Introduction}

[4+2] Cycloadditions of enolizable anhydrides (succinic IIa, glutaric IIb, or homophthalic IVa) and imines formed in situ or prepared in a separate step were discovered by Castagnoli and Cushman and have attracted considerable attention from chemists for the preparation of substituted $\gamma, \delta$, and $\varepsilon$-lactams (Scheme 1a) [1-3]. a) Previous work

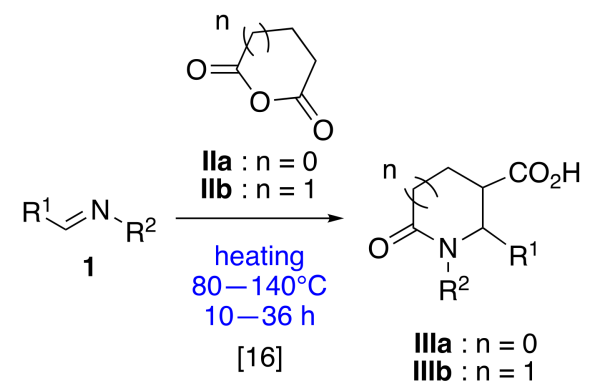

b) This work

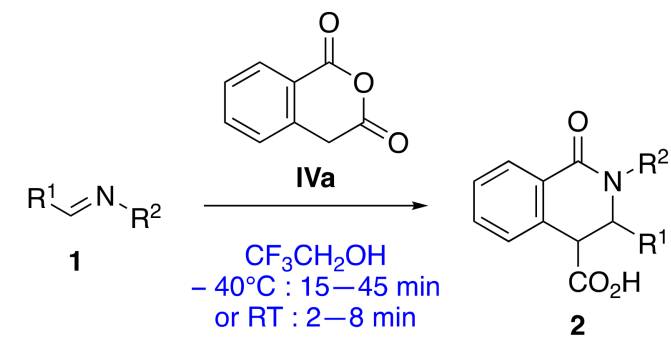

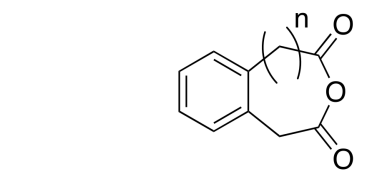

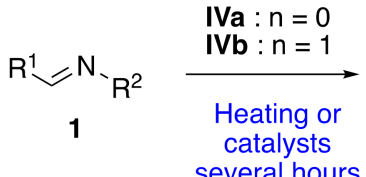

$[6,17-27]$

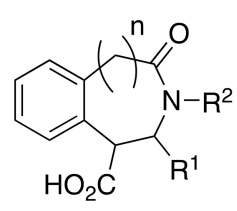

$\mathrm{Va}: \mathrm{n}=0$

Vb: $n=1$
Scheme 1. Castagnoli and Castagnoli-Cushman reactions.

Moreover, these cycloaddition reactions have seen wide applications in the synthesis of medicinal lead compounds piperidines and piperidinones derivatives [4,5], sting 
antagonist [6], and antimalarial [7] or anticancer agents [8-10]. In addition, lactams obtained by these reactions, and their reduced nitrogen heterocycle analogs are found to be key intermediates in the synthesis of biologically significant natural products such as 13-methyltetrahydroprotoberbines (i.e., (+)-thalictricavine, (+)-canadine, $(+)$ and (-)cavidine [11-13], phenanthridine alkaloids and $(-)$ and $(+)$-corynoline [14] possessing antitumor activity [15] (Figure 1).<smiles>CC(C)(C)c1ccc(N2C(=O)c3cc(F)ccc3[C@@H](CC(=O)O)[C@H]2c2ccc3c(c2)OCCO3)cc1Cl</smiles>

Sting antagonist<smiles>COc1ccc2c(c1OC)CN1CCc3cc4c(cc3[C@@H]2C1)OCO4</smiles>

(+)-Thalictricavine<smiles>N#Cc1cc(NC(=O)[C@@H]2CCC(=O)N(CC(F)(F)F)[C@H]2c2cccnc2)ccc1F</smiles>

Antimalarial properties<smiles>CN1Cc2c(ccc3c2OCO3)[C@@H]2[C@@H](O)Cc3cc4c(cc3[C@@H]21)OCO4</smiles>

(+)-Corynoline

Figure 1. Bioactive compounds and natural products.

Surprisingly, despite the high interest in this transformation, the intrinsic drawbacks associated with the Castagnoli-Cushman reaction, including low reaction rates, moderate diastereoselectivity, catalyst-free, thermal-free, and limited substrate scope, have not been solved yet.

Generally, the reaction of succinic IIa and glutaric Iib anhydrides with imines requires forcing conditions such as reflux in benzene, toluene, or $p$-xylene $\left(140{ }^{\circ} \mathrm{C}\right)$ during 12-36 $\mathrm{h}$ [16]. While the reaction conditions were less harsh when more enolizable anhydrides Iva and $\mathbf{I V b}$ were employed, catalysts or heating or long reactions time were necessary to isolate the CC products $\mathbf{V a}$ and $\mathbf{V b}$ in decent yields.

In addition, depending on the nature of the imine and the reaction conditions, the cycloaddition of homophthalic anhydride (HPA) IVa with imines affords the 3,4-cis product Va, 3,4-trans product Va, or a mixture of both predominantly [3].

Mixtures of 3,4-cis and 3,4-trans isomers have been observed in the presence of catalytic amounts of bases (DIEA, TEA), Lewis acids $\left(\mathrm{FeCl}_{3}, \mathrm{AlCl}_{3}, \mathrm{ZnCl}_{2}\right)$, protic acid ( $\left.\mathrm{HCl}\right)$, or in the absence of catalyst [3]. $\mathrm{BF}_{3}-\mathrm{OEt}_{2}$ [17], $\mathrm{TiCl}_{4}$ [18], $\mathrm{InCl}_{3}$ [6], and aspartic acid [19] have been employed for the preparation of 3,4-trans-isoquinolonic acids. Recently, 3,4trans-isoquinolonic acids have been prepared without catalyst by heating homophthalic anhydride IVa with different amines and aldehydes in the presence of $\mathrm{Na}_{2} \mathrm{SO}_{4}$ in refluxing toluene [20]. 3,4-cis-isoquinolonic acids have been prepared in the presence of ionic liquids [21], trimethyl orthoformate [22], $\mathrm{Yb}(\mathrm{OTf})_{3}$ [23], silica sulfuric acid [24], sulfonic acid functionalized silica [25], $\mathrm{KAl}\left(\mathrm{SO}_{4}\right)_{2} \cdot 12 \mathrm{H}_{2} \mathrm{O}$ [26], or L-proline [27]. The formations of cis-isoquinolonic acids under the conditions described above took several hours at room temperature or reflux in different solvents (Scheme 1a).

Despite the considerable attention that CCRs have received over the past decades, the development of mild and practical methods is an ongoing challenge in organic synthesis. Therefore, further exploration of new catalyst-free, mild, efficient, and fast conditions is still desirable to access substituted $\gamma, \delta$, and $\varepsilon$-lactams. 
Fluorinated alcohols may be considered promising alternatives to address this synthetic challenge due to their unique properties such as high dielectric constant, polarity, high $\mathrm{H}$-bond donor ability, and low nucleophilicity for cation stabilization [28,29].

Herein, we report a fast and powerful cycloaddition of imines 1 with HPA IVa in 2,2,2-trifluoroethanol (TFE), resulting in an efficient synthesis of lactams 2.2 are promising scaffolds for the design of biologically active compounds and key intermediates in the synthesis of natural products (Scheme 1b).

\section{Results and Discussion}

We initially investigated the reaction of HPA IVa with imine 1a in different solvents (Table 1).

Table 1. Optimization of the reaction conditions ${ }^{\mathrm{a}, \mathrm{b}}$.

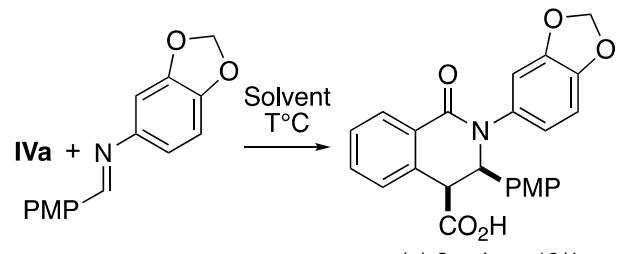

$1 a$

( \pm )-2a, d.r. $>19 / 1$

\begin{tabular}{ccccc}
\hline Entry & Solvent & Temp [ $\left.{ }^{\circ} \mathbf{C}\right]$ & Time & Yield $^{\mathbf{c}}$ \\
\hline 1 & $\mathrm{CH}_{2} \mathrm{Cl}_{2}$ & -40 & $24 \mathrm{~h}$ & 37 \\
2 & $\mathrm{CH}_{2} \mathrm{Cl}_{2}$ & $\mathrm{rt}$ & $16 \mathrm{~h}$ & 72 \\
3 & $\mathrm{toluene}$ & -40 & $20 \mathrm{~h}$ & 56 \\
4 & $\mathrm{ACN}$ & -40 & $20 \mathrm{~h}$ & 50 \\
5 & $\mathrm{MTBE}$ & -40 & $48 \mathrm{~h}$ & 78 \\
6 & $\mathrm{TFE}$ & -40 & $15 \mathrm{~min}$ & 71 \\
7 & $\mathrm{TFE}$ & $\mathrm{rt} \mathrm{min}$ & 32 \\
8 & $\mathrm{CH} \mathrm{Cl}_{2}$ & -40 & $3 \mathrm{~h}$ & 61 \\
9 & $(+0.1$ eq. TFE $)$ & 0 & $15 \mathrm{~min}$ & 52 \\
\hline
\end{tabular}

a General conditions: homophthalic anhydride IVa (1.5 eq.) and imine 1a (0.1 mmol). ${ }^{\mathrm{b}} \mathrm{PMP}=4-\mathrm{MeOC}_{6} \mathrm{H}_{4}$.

c Yield refer to chromatographically pure product.

This resulted in the formation of desired cis-lactam 2a with excellent diastereoselectivity (d.r. > 19:1). Interestingly, the reaction rate varied noticeably depending on the solvent and the temperature. In $\mathrm{CH}_{2} \mathrm{Cl}_{2}$, toluene, and $\mathrm{CH}_{3} \mathrm{CN}$, the reaction takes several hours at $-40{ }^{\circ} \mathrm{C}$, even at room temperature. In polar solvents such as $\mathrm{MTBE}$ at $-40{ }^{\circ} \mathrm{C}$, the reaction also takes several hours, but the yield is higher (78\%). To our delight, in TFE, the lactam 2a was obtained in $81 \%$ yield in $15 \mathrm{~min}$ at $-40{ }^{\circ} \mathrm{C}$ and in $72 \%$ yield in $2 \mathrm{~min}$ at room temperature (entries 6 and 7). Catalytic amounts of TFE in $\mathrm{CH}_{2} \mathrm{Cl}_{2}$ at $-40{ }^{\circ} \mathrm{C}$ lead to an 8 -fold decrease in reaction time (i.e., entries 1 and $8 ; 24 \mathrm{~h}$ vs. $3 \mathrm{~h}$ ) and an increase in yield (i.e., entries 1 and 8; 37\% vs. 61\%). Another fluorinated alcohol, hexafluoro-2-propanol (HFIP), could also be used, although the yield was lower compared with the result of TFE (i.e., entry 9). The reaction could not be performed at $-40{ }^{\circ} \mathrm{C}$ (solidifying of HFIP), and some degradation was observed at $0{ }^{\circ} \mathrm{C}$. The diastereomeric excess remains unchanged whatever the solvent. The substituents of the starting imine induce diastereoselectivity.

Different mechanisms have been proposed for the CCR, including an iminolysis pathway and a concerted [4+2] cycloaddition $[3,20]$. The most plausible mechanism for the reaction of homophthalic anhydride IVa with simple imines $\mathbf{1}$ in TFE is shown in Scheme 2, proposal mechanism 1 . The origin of the reaction's kinetic and yield increases in the presence of TFE might be attributed to a double activation of imine and carbonyl function of anhydride IVa via the H-bond network of TFE. The formation of hydrogen bonds between the anhydride and the TFE will decrease the $\mathrm{pK}_{\mathrm{a}}$ of the enolizable proton. This will facilitate the attack of the IVa enol on the imine carbon. In this case, one molecule of TFE can bind to both imine and the IVa enol. A probable concerted hydrogen transfer 
between activated imine and enol mediated by TFE could occur. This could explain the increase in reaction kinetics (Scheme 2). This scenario is supported by computational studies on closely related reactions of imines with $\alpha$-cyanosuccinic anhydride [5] and is consistent with the relatively high acidity of $\mathbf{I V a}\left(\mathrm{pK}_{\mathrm{a}}=8.15\right)$.

\section{Proposal mechanism :}

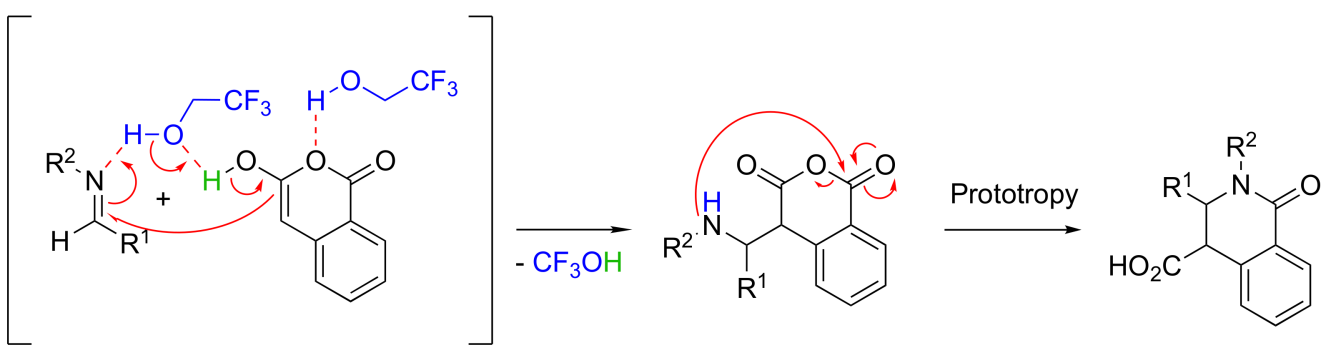

Intermediates in Perkin-type reaction

Scheme 2. Activation model via a putative transition state. $\mathrm{pK}_{\mathrm{a}}$ values: IVa $(8.15)$, imine $(\approx 10)$, TFE (12.37).

Interested in expanding the scope of the optimal conditions in hand, we next examine carboxylic acid anhydrides other than homophthalic anhydride IVa and a variety of imines (Figure 2). Imine 1a didn't react in TFE with glutaric anhydride, 3-methoxy-1H-isochromen1-one, 1,4-dioxane-2,6-dione and $(3 \mathrm{a} R, 7 \mathrm{a} S)$-hexahydroisobenzofuran-1,3-dione at $-40{ }^{\circ} \mathrm{C}$ $(24 \mathrm{~h})$ or at reflux $(24 \mathrm{~h})$ or under microwave irradiations at $150{ }^{\circ} \mathrm{C}(4 \mathrm{~h})$.

The scope of the reaction was found to be relatively broad. Imines tolerated different $\mathrm{N}$-aryl or $\mathrm{N}$-alkyl groups well. Imines derived from aromatic aldehydes bearing aromatic rings (such as phenol, protected phenol, PMP, p-nitrobenzene) were converted into the corresponding cycloadducts $\mathbf{2 b}, \mathbf{2 c}, \mathbf{2} \mathbf{d}, \mathbf{2 e}, \mathbf{2} \mathbf{i}$, and $\mathbf{2 h}$ in good yields (74-97\%) with excellent diastereoselectivity except for $\mathbf{2 h}$. Imines bearing alkyl groups (such as $\mathrm{N}$-tert-butyl or C-isopropyl) were also converted into the corresponding cycloadducts $\mathbf{2 e}(79 \%)$ and $\mathbf{2 f}$ $(40 \%)$ with excellent diastereoselectivity. The yield of $\mathbf{2} \mathbf{f}$ is lower because the imine $\mathbf{1 f}$ is unstable. Imine $\mathbf{1 g}$ derived from heterocyclic aldehyde (i.e., thiophene-2-carbaldehyde) also performed very well (i.e., $\mathbf{2 g}$, 90\%), although the diastereoselectivity was reduced. The presence of an electron-withdrawing group such as a C-ethylcarboxylate on the imine (i.e., 1j) led to $2 \mathbf{j}$ but in lower yield (68\%) with excellent diastereoselectivity.

The best yields have been obtained at room temperature. The diastereoselectivity was the same at $-40{ }^{\circ} \mathrm{C}$ or room temperature for $\mathbf{2 b}, \mathbf{2} \mathbf{c}, \mathbf{2 e}$, and $\mathbf{2} \mathbf{f}$, whereas it changed for $\mathbf{2 d}$. The lower d.r. value for $\mathbf{2} \mathbf{d}$ at room temperature may be due to the flexibility of the $\mathrm{CH}_{2}-$ PMP group attached to the nitrogen atom of the imine. The formation of cis-diastereoisomer was observed in all cases except for $\mathbf{2 h}$ and $\mathbf{2 j}$. The trans-diastereoisomer was major for $\mathbf{2 h}$. $2 \mathbf{j}$ was obtained as trans-diastereoisomer solely.

The group's properties linked to the imine's carbon may explain these results. The presence of electron-rich groups promotes the formation of the cis-diastereoisomer, whereas electron-poor groups promote the synthesis of the trans-diastereoisomer. An ethylcarboxylate function being more electron-withdrawing than nitrobenzene may explain the exclusive formation of the trans-diastereoisomer $\mathbf{2} \mathbf{j}$.

The CCR usually leads to the kinetic cis-diastereoisomer product without catalyst or adduct. The thermodynamic trans-diastereoisomer can be obtained by epimerization in good yield upon exposure to DBU [30]. The cis-3,4-lactam 2a was epimerized in trans-3,4lactam $\mathbf{2} \mathbf{a}^{\prime}$ in $\mathbf{8 5} \%$ yield without loss of diastereoselectivity (Scheme 3). 


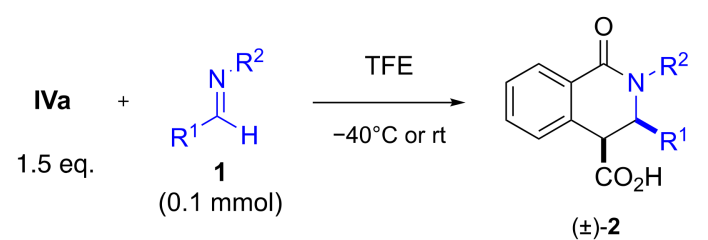<smiles>O=C(O)C1c2ccccc2C(=O)N(c2ccc3c(c2)OCO3)C1c1ccc(O)cc1</smiles>

$( \pm)-2 b$<smiles>O=C(O)Cc1ccc(C2C(C(=O)OCc3ccccc3)c3ccccc3C(=O)N2c2ccc3c(c2)OCO3)cc1</smiles>

$( \pm)-2 c$<smiles>NPC1C(C(=O)O)c2ccccc2C(=O)N1CC(F)(F)F</smiles>

$( \pm)-2 d$

$63 \%\left(20 \mathrm{~min},-40^{\circ} \mathrm{C}\right)$, d.r. $>19 / 1$ $97 \%$ (2 min, rt), d.r. : $2.2 / 1$<smiles>CC(C)(C)N1C(=O)c2ccccc2C(C(=O)O)C1[18OH]</smiles><smiles>CC(C)C1C(C(=O)O)c2ccccc2C(=O)N1c1ccccc1</smiles>

$( \pm)-2 \mathrm{e}$
$69 \%\left(40 \mathrm{~min},-40^{\circ} \mathrm{C}\right)$, d.r. $>19 / 141 \%\left(40 \mathrm{~min},-40^{\circ} \mathrm{C}\right)$, d.r. $>19 / 1$ $79 \%$ (2 min, rt), d.r. $>19 / 1$ $40 \%$ (5 min, rt), d.r. $>19 / 1$<smiles>CCCCN1C(=O)c2ccccc2[C@H](C(=O)O)C1c1ccc([N+](=O)[O-])cc1</smiles>

$( \pm)-2 h$<smiles>NP(F)(F)(F)C1C(C(=O)O)c2ccccc2C(=O)N1c1ccc(C(F)(F)F)cc1</smiles>

$( \pm)-2 \mathbf{i}$

$77 \%$ (2 min, rt), d.r. : $1.6 / 1$<smiles>O=C(O)C1c2ccccc2C(=O)N(C2CC2)C1c1cccs1</smiles>

$( \pm)-2 g$

$90 \%(8 \mathrm{~min}, \mathrm{rt})$, d.r. : $1.8 / 1$<smiles>CCOC(=O)C1C(C(=O)O)c2ccccc2C(=O)N1[P+]</smiles>

$( \pm)-2 \mathbf{j}$ $68 \%(8 \mathrm{~min}$, rt), d.r. $>19 / 1$

Figure 2. Substrate scope of imines 1. d.r. was determined by ${ }^{1} \mathrm{H}-\mathrm{NMR}$ Yields referred to pure products. The formation of $\mathbf{2} \mathbf{g}-\mathbf{2} \mathbf{j}$ products was carried out only at room temperature.

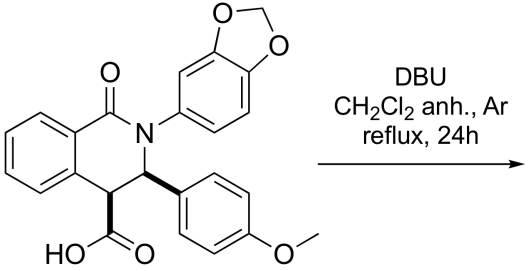

$( \pm)$-cis<smiles>COc1ccc([C@H]2[C@@H](C(=O)O)c3ccccc3C(=O)N2c2ccc3c(c2)OCO3)cc1</smiles>

$( \pm)$-trans

2a, d.r. $>19 / 1$

2a', d.r. $>19 / 1$

Scheme 3. Epimerization of compound 2a in $2 \mathbf{a}^{\prime}$.

\section{Materials and Methods}

\subsection{General Information}

Reagents and solvents were purchased from commercial sources and were purified by distillation or recrystallization prior to use. Reactions were run under an argon atmosphere unless stated otherwise. The purification of reaction products was carried out by flash column chromatography using silica gel $\left(60 \mathrm{~F}_{254}\right)$ packed Redisep or Interchim columns (230-400 mesh). Preparative thin-layer chromatography was performed on MachereyNagel $0.25 \mathrm{~mm}$ silica gel $\left(60 \mathrm{~F}_{254}\right)$ glass plates. Melting points were recorded on a B540 Büchi melting point apparatus and are uncorrected. Infrared spectra were recorded on a Perkin Elmer FT-IR 100 spectrum spectrometer. Proton nuclear magnetic resonance 
spectra $\left({ }^{1} \mathrm{H}-\mathrm{NMR}\right)$ were recorded on Bruker Advance-300 or Bruker AC-500 machines and are reported in parts per million (ppm) using solvent as an internal standard $\left(\mathrm{CDCl}_{3}\right.$ at $7.26 \mathrm{ppm}, \mathrm{DMSO}-\mathrm{d}_{6}$ at $2.50 \mathrm{ppm}$, or $\mathrm{CD}_{3} \mathrm{OD}$ at $\left.3.31 \mathrm{ppm}\right)$. For ${ }^{1} \mathrm{H} \mathrm{NMR}$, the NMR spectroscopic data are given in parts per million (ppm). Coupling constants, usually denoted $J$, are given in the unit of Hertz $(\mathrm{Hz})$. Multiplicities are designed by abbreviation: singlet (s), broad singlet (bs), doublet (d), doublet of doublet (dd), doublet of doublet of doublet (ddd), triplet (t), doublet of triplet (d), quartet (q), quintet (quint.), multiplet $(\mathrm{m})$, etc. Proton-decoupled carbon nuclear magnetic resonance spectra $\left({ }^{13} \mathrm{C}-\mathrm{NMR}\right)$ were recorded on Bruker Advance-300 or Bruker AC-500 machines and are reported in parts per million (ppm) using solvent as an internal standard $\left(\mathrm{CDCl}_{3}\right.$ at $77.1 \mathrm{ppm}, \mathrm{DMSO}-\mathrm{d}_{6}$ at $39.5 \mathrm{ppm}$, or $\mathrm{CD}_{3} \mathrm{OD}$ at $49.0 \mathrm{ppm}$ ). The d.r. was calculated with ${ }^{1} \mathrm{H} \mathrm{NMR}$. Mass data were obtained on an AUTOMASS ThermoFinnigan spectrometer with electrospray or electronebullization ionization and quadrupole mass filter. HRMS data were recorded either by LCT spectrometer (Waters) or LCT Premier XE (Waters) with ESI ionization and TOF analyzer. Reactions under microwave irradiation were performed in an Anton Parr MCP300 reactor.

\subsection{Synthesis}

General Procedure for Castagnoli-Cushman reaction (2a-2f). A round-bottom flask under an argon atmosphere was charged with the corresponding imine $\mathbf{1}(0.100 \mathrm{mmol}$, 1.0 eq.) in TFE ( $3 \mathrm{~mL})$ and then charged with homophthalic anhydride IVa $(24.3 \mathrm{mg}$, $0.150 \mathrm{mmol}, 1.5$ eq.) at $-40{ }^{\circ} \mathrm{C}$. The reaction mixture was stirred at $-40{ }^{\circ} \mathrm{C}$ until the starting material was consumed, as indicated by TLC analysis. The mixture was concentrated in vacuo, and the residue was purified by preparative TLC to afford the pure product. Purification: $\mathrm{CH}_{2} \mathrm{Cl}_{2} / \mathrm{MeOH}$ (95:5). Copies of ${ }^{1} \mathrm{H}$ and ${ }^{13} \mathrm{C}$ spectra for all prepared are available in the supplementary materials.

4-(benzyloxy)benzaldehyde (0c): The title compound was prepared following the literature procedure [31] using $p$-anisaldehyde (2.30 g, $18.846 \mathrm{mmol}, 1.0 \mathrm{eq}$.) and benzyl bromide (3.36 mL, $28.269 \mathrm{mmol}, 1.5$ eq.). Product 0c was isolated in 93\% overall yield (3.74 g) in one step as a white powder. Mp: $68-70{ }^{\circ} \mathrm{C}$; IR (v): 1738, 1604, 1507, 1451, 1266, 1061, 708, $617 \mathrm{~cm}^{-1} ;{ }^{1} \mathrm{H}$ NMR (300 MHz, $\left.\mathrm{CDCl}_{3}\right)$ : d (ppm) $9.91(\mathrm{~s}, 1 \mathrm{H}), 7.87(\mathrm{~d}, J=8.9 \mathrm{~Hz}, 2 \mathrm{H})$, 7.48-7.37 (m, 5H), $7.10(\mathrm{~d}, J=8.9 \mathrm{~Hz}, 2 \mathrm{H}), 5.18(\mathrm{~s}, 2 \mathrm{H}) ;{ }^{13} \mathrm{C}$ NMR $\left(125 \mathrm{MHz}, \mathrm{CDCl}_{3}\right)$ : d (ppm) 190.8, 163.7, 136.0, 132.0, 130.1, 128.7, 128.3, 127.5, 115.1, 70.2; HRMS (E.S.I. ${ }^{+}, \mathrm{m} / \mathrm{z}$ ) calcd for $\mathrm{C}_{14} \mathrm{H}_{13} \mathrm{O}_{2}{ }^{+}(\mathrm{M}+\mathrm{H})^{+}:$213.0910, found: 213.0887 .

$N$-(benzo[d][1,3]dioxol-5-yl)-1-(4-methoxy phenyl)methanimine (1a): To a stirred solution of benzo[d][1,3]dioxol-5-amine (1.28 g, $9.326 \mathrm{mmol}, 1.0$ eq.) in dry MeOH (40 mL) with molecular sieve $4 \AA$ were added $p$-anisaldehyde $(1.13 \mathrm{~mL}, 9.326 \mathrm{mmol}, 1.0$ eq.) and glacial acetic acid $(53.4 \mu \mathrm{L}, 0.933 \mathrm{mmol}, 0.1 \mathrm{eq}$.$) . The reaction mixture was stirred overnight at$ reflux. After cooling, the mixture was filtered on celite and concentrated under reduced pressure. The residue was purified by recrystallisation (heptane/AcOEt, 95:5) to afford the pure product 1a. Brown crystals $(1.71 \mathrm{~g}, 72 \%)$. Mp: $107-108{ }^{\circ} \mathrm{C}$; IR ( $\left.v\right): 2889,1601$, 1574, 1499, 1479, 1241, 1170, 1028, 924, $816 \mathrm{~cm}^{-1} ;{ }^{1} \mathrm{H}$ NMR (500 MHz, CDCl $)$ : d (ppm) 8.30 (s, 1H), $7.75(\mathrm{~d}, J=8.7 \mathrm{~Hz}, 2 \mathrm{H}), 6.90(\mathrm{~d}, J=8.7 \mathrm{~Hz}, 2 \mathrm{H}), 6.74(\mathrm{~d}, J=8.3 \mathrm{~Hz}, 1 \mathrm{H}), 6.73(\mathrm{~d}$, $J=2.1 \mathrm{~Hz}, 1 \mathrm{H}), 6.65(\mathrm{dd}, J=8.3 \mathrm{~Hz}, J=2.1 \mathrm{~Hz}, 1 \mathrm{H}), 5.90(\mathrm{~s}, 2 \mathrm{H}), 3.79(\mathrm{~s}, 3 \mathrm{H}) ;{ }^{13} \mathrm{C} \mathrm{NMR}$ $\left(125 \mathrm{MHz} \mathrm{CDCl}_{3}\right)$ : d (ppm) 162.1, 158.1, 148.2, 146.9, 145.8, 130.3 (2C), 129.3, 114.6, 114.2 (2C), 108.3, 101.9, 101.3, 55.4; HRMS (E.S.I. ${ }^{+}, \mathrm{m} / \mathrm{z}$ ) calcd for $\mathrm{C}_{15} \mathrm{H}_{14} \mathrm{NO}_{3}{ }^{+}(\mathrm{M}+\mathrm{H})^{+}: 256.0968$, found: 256.0955 .

4-((benzo[d][1,3]dioxol-5-ylimino)methyl) phenol (1b): To a stirred solution of benzo[d][1,3]dioxol-5-amine (13.7 mg, $0.100 \mathrm{mmol}, 1.0$ eq.) in dry $\mathrm{MeOH}(2 \mathrm{~mL})$ with molecular sieve $4 \AA$ were added 4-hydroxybenzaldehyde $(10.0 \mu \mathrm{L}, 0.100 \mathrm{mmol}, 1.0$ eq.) and one drop of glacial acetic acid $(0.010 \mathrm{mmol}, 0.1 \mathrm{eq}$.$) . The reaction mixture was stirred$ overnight at reflux. After cooling, the mixture was concentrated under reduced pressure to afford the brut product $\mathbf{1 b}$ without further purification. The imine was used in Castagnoli-Cushman reactions directly after preparation 
$N$-(benzo[d][1,3]dioxol-5-yl)-1-(4-(benzyloxy) phenyl)methanimine (1c): To a stirred solution of benzo[d][1,3]dioxol-5-amine (193.0 mg, $1.407 \mathrm{mmol}, 1.0$ eq.) in dry $\mathrm{MeOH}(7 \mathrm{~mL})$ with molecular sieve $4 \AA$ were added aldehyde $0 \mathrm{c}(298.6 \mathrm{mg}, 1.407 \mathrm{mmol}, 1.0$ eq. $)$ and glacial acetic acid $(8.1 \mu \mathrm{L}, 0.141 \mathrm{mmol}, 0.1 \mathrm{eq}$.). The reaction mixture was stirred for $1 \mathrm{~h}$ at reflux. After cooling, the mixture was filtered on celite and concentrated under reduced pressure to afford the pure product 1c. Brown solid (462.8 mg, quant.). Mp: $102-104{ }^{\circ} \mathrm{C}$; IR (v): 2962, 2922, 1605, 1571, 1506, 1479, 1257, 1085, 1012, $791 \mathrm{~cm}^{-1}$; ${ }^{1} \mathrm{H}$ NMR (500 MHz, DMSO- $d_{6}$ ): d (ppm) $8.53(\mathrm{~s}, 1 \mathrm{H}), 7.85(\mathrm{~d}, J=8.7 \mathrm{~Hz}, 2 \mathrm{H}), 7.49-7.32(\mathrm{~m}, 5 \mathrm{H}), 7.13(\mathrm{~d}, J=8.7 \mathrm{~Hz}, 2 \mathrm{H})$, $6.96(\mathrm{~d}, J=2.1 \mathrm{~Hz}, 1 \mathrm{H}), 6.92(\mathrm{~d}, J=8.1 \mathrm{~Hz}, 1 \mathrm{H}), 6.76(\mathrm{dd}, J=8.1 \mathrm{~Hz}, J=2.1 \mathrm{~Hz}, 1 \mathrm{H}), 6.04(\mathrm{~s}$, 2H), $5.19(\mathrm{~s}, 2 \mathrm{H}) ;{ }^{13} \mathrm{C}$ NMR (125 MHz, DMSO- $\left.d_{6}\right): \mathrm{d}(\mathrm{ppm}) 160.7,158.1,147.9,146.0,145.4$, 136.7, 130.1 (2C), 129.2, 128.4 (2C), 127.9, 127.7 (2C), 115.2, 115.0 (2C), 108.4, 101.4, 101.2, 69.4; HRMS (E.S.I. ${ }^{+}, \mathrm{m} / \mathrm{z}$ ) calcd for $\mathrm{C}_{21} \mathrm{H}_{18} \mathrm{NO}_{3}{ }^{+}(\mathrm{M}+\mathrm{H})^{+}: 332.1281$, found: 322.0998 .

$N$-(4-methoxybenzyl)-1-(4-methoxyphenyl) methanimine (1d): The title compound was prepared following the literature procedure [32] using (4-methoxyphenyl)methanamine (620.0 $\mu \mathrm{L}, 4.746 \mathrm{mmol}, 1.0$ eq.) and $p$-anisaldehyde ( $577.5 \mu \mathrm{L}, 4.746 \mathrm{mmol}, 1.0$ eq.). Product $1 \mathrm{~d}$ was isolated in quant. overall yield $(1.20 \mathrm{~g})$ in one step as a brown solid. Mp: $36-37^{\circ} \mathrm{C}$; IR (v): 2830, 2910, 1510, 1245, 1035, $810 \mathrm{~cm}^{-1} ;{ }^{1} \mathrm{H} \mathrm{NMR}\left(300 \mathrm{MHz}, \mathrm{CDCl}_{3}\right)$ : d (ppm) 8.32 $(\mathrm{s}, 1 \mathrm{H}), 7.73(\mathrm{~d}, J=8.8 \mathrm{~Hz}, 2 \mathrm{H}), 7.27(\mathrm{~d}, J=8.8 \mathrm{~Hz}, 2 \mathrm{H}), 6.95(\mathrm{~d}, J=8.7 \mathrm{~Hz}, 2 \mathrm{H}), 6.91(\mathrm{~d}$, $J=8.7 \mathrm{~Hz}, 2 \mathrm{H}), 4.75(\mathrm{~s}, 2 \mathrm{H}), 3.85(\mathrm{~s}, 3 \mathrm{H}), 3.81(\mathrm{~s}, 3 \mathrm{H}) ;{ }^{13} \mathrm{C} \mathrm{NMR}\left(75 \mathrm{MHz}, \mathrm{CDCl}_{3}\right): \mathrm{d}(\mathrm{ppm})$ 161.7, 160.9, 158.7, 131.7, 129.8 (2C), 129.2 (2C), 114.0 (2C), 113.9 (2C), 64.4, 55.3, 55.2; HRMS (E.S.I. ${ }^{+}, \mathrm{m} / \mathrm{z}$ ) calcd for $\mathrm{C}_{16} \mathrm{H}_{18} \mathrm{NO}_{2}{ }^{+}(\mathrm{M}+\mathrm{H})^{+}$: 256.1332, found: 256.1342 .

N-tert-butyl-1-(4-methoxyphenyl)methanimine (1e): To a stirred solution of tert-butylamine $(10.7 \mu \mathrm{L}, 0.100 \mathrm{mmol}, 1.0$ eq. $)$ in dry MTBE $(2 \mathrm{~mL})$ with molecular sieve $4 \AA$ was added $p$-anisaldehyde $(12.2 \mu \mathrm{L}, 0.100 \mathrm{mmol}, 1.0$ eq.). The reaction mixture was stirred for $24 \mathrm{~h}$ at room temperature. The mixture was concentrated under reduced pressure to afford the brut product 1e without further purification. The imine was used in Castagnoli-Cushman reactions directly after preparation.

2-methyl-N-phenylpropan-1-imine (1f): To a stirred solution of aniline $(9.1 \mu \mathrm{L}, 0.100 \mathrm{mmol}$, 1.0 eq.) in dry $\mathrm{CH}_{2} \mathrm{Cl}_{2}(1 \mathrm{~mL})$ with $\mathrm{MgSO}_{4}(18.1 \mathrm{mg}, 0.150 \mathrm{mmol}, 1.5 \mathrm{eq})$ was added isobutyraldehyde $(13.7 \mu \mathrm{L}, 0.150 \mathrm{mmol}, 1.5$ eq.). The reaction mixture was stirred for $1 \mathrm{~h}$ at reflux. After cooling, the mixture was filtered and concentrated under reduced pressure to afford the brut product $\mathbf{1 f}$ without further purification. The imine was used in Castagnoli-Cushman reactions directly after preparation.

N-cyclopropyl-1-(thiophen-2-yl)methanimine (1g): The title compound was prepared following the literature procedure [33] using cyclopropanamine $(6.9 \mu \mathrm{L}, 0.100 \mathrm{mmol}, 1.0 \mathrm{eq}$.) and thiophene-2-carbaldehyde $(9.4 \mu \mathrm{L}, 0.100 \mathrm{mmol}, 1.0 \mathrm{eq}$.). The brut product $1 \mathrm{~g}$ was used in Castagnoli-Cushman reactions without further purification directly after preparation. The imine was used in Castagnoli-Cushman reactions directly after preparation.

N-butyl-1-(4-nitrophenyl)methanimine (1h): To a stirred solution of butan-1-amine $(9.9 \mu \mathrm{L}$, $0.100 \mathrm{mmol}, 1.0$ eq.) in dry toluene $(1 \mathrm{~mL})$ with $\mathrm{MgSO}_{4}(18.1 \mathrm{mg}, 0.150 \mathrm{mmol}, 1.5 \mathrm{eq})$ was added 4-nitrobenzaldehyde ( $15.1 \mathrm{mg}, 0.100 \mathrm{mmol}, 1.0 \mathrm{eq}$.). The reaction mixture was stirred overnight at $80^{\circ} \mathrm{C}$. After cooling, the mixture was filtered and concentrated under reduced pressure to afford the brut product $1 \mathrm{~h}$ without further purification. The imine was used in Castagnoli-Cushman reactions directly after preparation.

1-(4-methoxyphenyl)-N-(4-(trifluoromethyl) phenyl)methanimine (1i): To a stirred solution of 4-(trifluoromethyl)aniline (12.6 $\mu \mathrm{L}, 0.100 \mathrm{mmol}, 1.0$ eq.) in dry $\mathrm{MeOH}(1 \mathrm{~mL})$ with molecular sieve $4 \AA$ were added 4-methoxybenzaldehyde $(12.2 \mu \mathrm{L}, 0.100 \mathrm{mmol}, 1.0$ eq.) and one drop of glacial acetic acid $(0.010 \mathrm{mmol}, 0.1 \mathrm{eq}$.$) . The reaction mixture was stirred$ overnight at reflux. After cooling, the mixture was filtered and concentrated under reduced pressure to afford the brut product $1 \mathbf{i}$ without further purification. The imine was used in Castagnoli-Cushman reactions directly after preparation.

Ethyl 2-((4-methoxyphenyl)imino)acetate (1j): The title compound was prepared following the literature procedure [34] using 4-methoxyaniline (12.3 mg, $0.100 \mathrm{mmol}, 1.0$ eq.) and ethyl 2-oxoacetate $(20.4 \mu \mathrm{L}, 0.100 \mathrm{mmol}, 1.0$ eq., $50 \%$ in toluene). The brut product $1 \mathbf{j}$ was 
used without further purification. The imine was used in Castagnoli-Cushman reactions directly after preparation.

Cis-2-(benzo[d][1,3]dioxol-5-yl)-3-(4-metho xyphenyl)-1-oxo-1,2,3,4-tetrahydroisoquinoline4-carboxylic acid (2a): The title compound was prepared following the general procedure described above using $\mathbf{1 a}(25.5 \mathrm{mg}, 0.100 \mathrm{mmol}, 1.0$ eq.) and IVa (24.3 $\mathrm{mg}, 0.150 \mathrm{mmol}$, 1.5 eq.) in 15 min of reaction time. The product 2 a was isolated in $81 \%$ yield (33.8 $\mathrm{mg}$, d.r. (cis/trans): $>19 / 1$ ) as a brown powder. When the reaction was performed at room temperature in $2 \mathrm{~min}$ of reaction time: $30.1 \mathrm{mg}, 72 \%$, d.r. (cis/trans): $>19 / 1$. Mp: $158-159{ }^{\circ} \mathrm{C}$; IR ( $v): 3321,2902,1717,1609,1485,1246,1175,1032,926,796,731 \mathrm{~cm}^{-1}$; ${ }^{1} \mathrm{H}$ NMR $(300 \mathrm{MHz}$, $\left.\mathrm{CDCl}_{3}\right): \mathrm{d}(\mathrm{ppm}) 8.30(\mathrm{dd}, J=7.0 \mathrm{~Hz}, J=1.2 \mathrm{~Hz}, 1 \mathrm{H}), 7.60-7.48(\mathrm{~m}, 3 \mathrm{H}), 6.97(\mathrm{dd}, J=6.7 \mathrm{~Hz}$, $J=2.0 \mathrm{~Hz}, 2 \mathrm{H}), 6.75(\mathrm{~d}, J=8.0 \mathrm{~Hz}, 1 \mathrm{H}), 6.70(\mathrm{dd}, J=6.8 \mathrm{~Hz}, J=2.0 \mathrm{~Hz}, 2 \mathrm{H}), 6.63(\mathrm{~d}, J=2.0$ $\mathrm{Hz}, 1 \mathrm{H}), 6.59(\mathrm{dd}, J=8.0 \mathrm{~Hz}, J=2.0 \mathrm{~Hz}, 1 \mathrm{H}), 5.96(\mathrm{~s}, 2 \mathrm{H}), 5.28(\mathrm{~d}, J=6.0 \mathrm{~Hz}, 1 \mathrm{H}), 4.92(\mathrm{~d}$, $J=6.0 \mathrm{~Hz}, 1 \mathrm{H}), 3.74(\mathrm{~s}, 3 \mathrm{H}) ;{ }^{13} \mathrm{C} \mathrm{NMR}\left(125 \mathrm{MHz}, \mathrm{CDCl}_{3}\right): \mathrm{d}(\mathrm{ppm}) 172.0,163.9,159.7,147.8$, $146.7,135.4,132.6,132.5,129.2,129.1$ (2C), 128.8, 128.3, 128.1, 127.7, 120.6, 113.9 (2C), 108.8, 108.2, 101.5, 65.3, 55.1, 49.5; HRMS (E.S.I. ${ }^{+}, \mathrm{m} / \mathrm{z}$ ) calcd for $\mathrm{C}_{24} \mathrm{H}_{20} \mathrm{NO}_{6}{ }^{+}(\mathrm{M}+\mathrm{H})^{+}: 418.1285$, found: 418.1352 .

Trans-2-(benzo[d][1,3]dioxol-5-yl)-3-(4-methoxyphenyl)-1-oxo-1,2,3,4-tetrahydroisoquinoline4-carboxylic acid $\left(2 a^{\prime}\right)$ : A round-bottom flask under an argon atmosphere was charged with 2a (41.7 mg, $0.100 \mathrm{mmol}, 1 \mathrm{eq})$ in dry $\mathrm{CH}_{2} \mathrm{Cl}_{2}(2 \mathrm{~mL})$, and DBU (5.6 $\left.\mu \mathrm{L}, 0.050 \mathrm{mmol}, 0.5 \mathrm{eq}\right)$ was added. The reaction mixture was stirred for $24 \mathrm{~h}$ at reflux. The resultant solution was concentrated under reduced pressure, and the residue was purified by preparative TLC to afford the pure product 2a'. Purification: $\mathrm{CH}_{2} \mathrm{Cl}_{2} / \mathrm{MeOH}(95 / 5)$. White amorphous solid (35.5 mg, 85\%), d.r. (trans/cis) >19/1. IR (v): 3321, 2902, 1717, 1609, 1485, 1246, $1175,1032,926,796,731 \mathrm{~cm}^{-1} ;{ }^{1} \mathrm{H}$ NMR $\left(300 \mathrm{MHz}, \mathrm{CDCl}_{3}\right): \delta(\mathrm{ppm}) 8.18(\mathrm{dd}, J=6.5 \mathrm{~Hz}$, $J=2.7 \mathrm{~Hz}, 1 \mathrm{H}), 7.42(\mathrm{dd}, J=6.5 \mathrm{~Hz}, J=5.4 \mathrm{~Hz}, 2 \mathrm{H}), 7.22(\mathrm{dd}, J=5.4 \mathrm{~Hz}, J=2.7 \mathrm{~Hz}, 1 \mathrm{H}), 7.00(\mathrm{~d}$, $J=8.6 \mathrm{~Hz}, 2 \mathrm{H}), 6.84(\mathrm{~d}, J=1.7 \mathrm{~Hz}, 1 \mathrm{H}), 6.76(\mathrm{dd}, J=8.3 \mathrm{~Hz}, J=1.7 \mathrm{~Hz}, 1 \mathrm{H}), 6.72(\mathrm{~d}$, $J=8.6 \mathrm{~Hz}, 2 \mathrm{H}), 6.69(\mathrm{~d}, J=8.3 \mathrm{~Hz}, 1 \mathrm{H}), 5.90(\mathrm{~d}, J=1.4 \mathrm{~Hz}, 2 \mathrm{H}), 5.49(\mathrm{~s}, 1 \mathrm{H}), 3.94(\mathrm{~s}, 1 \mathrm{H}), 3.72$ (s, 3H); ${ }^{13} \mathrm{C}$ NMR $\left(75 \mathrm{MHz}, \mathrm{CDCl}_{3}\right): \delta$ (ppm) 173.2, 163.6, 159.3, 147.7, 146.5, 136.1, 132.5, $132.4,130.8,129.6,129.3,128.5,128.4,127.6$ (2C), 120.3, 114.1 (2C), 108.6, 108.2, 101.4, 64.7, 55.2, 53.4; (E.S.I. ${ }^{+}, \mathrm{m} / \mathrm{z}$ ) calcd for $\mathrm{C}_{24} \mathrm{H}_{20} \mathrm{NO}_{6}{ }^{+}(\mathrm{M}+\mathrm{H})^{+}: 418.1285$, found: 418.1352 .

Cis-2-(benzo[d][1,3]dioxol-5-yl)-3-(4-hydroxy phenyl)-1-oxo-1,2,3,4-tetrahydroisoquinoline4-carboxylic acid $(\mathbf{2 b})$ : The title compound was prepared following the general procedure described above using the brut $\mathbf{1 b}(0.100 \mathrm{mmol}, 1.0$ eq. $)$ and IVa $(24.3 \mathrm{mg}, 0.150 \mathrm{mmol}$, 1.5 eq.) in 20 min of reaction time. The product $2 \mathbf{b}$ was isolated in $71 \%$ yield $(28.6 \mathrm{mg}$, d.r. (cis/trans): 15.5/1) as a beige powder. When the reaction was performed at room temperature in $2 \mathrm{~min}$ of reaction time: $29.9 \mathrm{mg}, 74 \%$, d.r. (cis/trans): 15.5/1. Mp: 205-206 ${ }^{\circ} \mathrm{C}$; IR (v): 3389, 2904, 1717, 1591, 1557, 1513, 1483, 1433, 1249, 1197, 1175, 1034, 927, 786, 703 $\mathrm{cm}^{-1} ;{ }^{1} \mathrm{H}$ NMR (300 MHz, CD $\left.\mathrm{CD}_{3} \mathrm{OD}\right) \mathrm{d}(\mathrm{ppm}) 8.14(\mathrm{dd}, J=7.6 \mathrm{~Hz}, J=1.1 \mathrm{~Hz}, 1 \mathrm{H}), 7.68(\mathrm{~d}$, $J=7.6 \mathrm{~Hz}, 1 \mathrm{H}), 7.58(\mathrm{td}, J=7.5 \mathrm{~Hz}, J=1.1 \mathrm{~Hz}, 1 \mathrm{H}), 7.49(\mathrm{t}, J=7.5 \mathrm{~Hz}, 1 \mathrm{H}), 6.88(\mathrm{~d}$, $J=8.5 \mathrm{~Hz}, 2 \mathrm{H}), 6.75(\mathrm{~d}, J=8.9 \mathrm{~Hz}, 1 \mathrm{H}), 6.62(\mathrm{~d}, J=8.9 \mathrm{~Hz}, 2 \mathrm{H}), 6.57(\mathrm{~d}, J=8.5 \mathrm{~Hz}, 2 \mathrm{H}), 5.94(\mathrm{~d}$, $J=2.8 \mathrm{~Hz}, 2 \mathrm{H}), 5.31(\mathrm{~d}, J=5.9 \mathrm{~Hz}, 1 \mathrm{H}), 4.82(\mathrm{~d}, J=5.9 \mathrm{~Hz}, 1 \mathrm{H}) ;{ }^{13} \mathrm{C} \mathrm{NMR}\left(75 \mathrm{MHz}, \mathrm{CD}_{3} \mathrm{OD}\right)$ : d (ppm) 172.7, 166.3, 158.7, 149.2, 148.2, 136.6, 136.2, 133.8, 130.7 (2C), 130.3, 129.3, 129.2, 128.8, 122.2, 116.4, 116.0 (2C), 109.9, 108.9, 102.9, 67.0, 51.0; HRMS (E.S.I. ${ }^{+}, \mathrm{m} / \mathrm{z}$ ) calcd for $\mathrm{C}_{23} \mathrm{H}_{18} \mathrm{NO}_{6}{ }^{+}(\mathrm{M}+\mathrm{H})^{+}:$404.1129, found: 404.1090 .

Cis-2-(benzo[d][1,3]dioxol-5-yl)-3-(4-(benzyloxy)phenyl)-1-oxo-1,2,3,4tetrahydroisoquinoline -4-carboxylic acid (2c): The title compound was prepared following the general procedure described above using 1c (33.1 mg, $0.100 \mathrm{mmol}, 1.0$ eq.) and IVa (24.3 mg, $0.150 \mathrm{mmol}$, 1.5 eq.) in $45 \mathrm{~min}$ of reaction time. The product $2 \mathrm{c}$ was isolated in $72 \%$ yield $(35.5 \mathrm{mg}$, d.r. (cis/trans): 13/1) as a brown powder. When the reaction was performed at room temperature in $8 \mathrm{~min}$ of reaction time: $40.0 \mathrm{mg}, 81 \%$, d.r. (cis/trans): 13/1. Mp: 177$178{ }^{\circ} \mathrm{C}$; IR $(v): 3289,3059,1715,1644,1600,1504,1487,1384,1246,1036,1007,792,733$, $699 \mathrm{~cm}^{-1}$; ${ }^{1} \mathrm{H}$ NMR (300 MHz, CD $\left.\mathrm{OD}\right): \mathrm{d}(\mathrm{ppm}) 8.08(\mathrm{dd}, J=7.7 \mathrm{~Hz}, J=1.3 \mathrm{~Hz}, 1 \mathrm{H}), 7.78(\mathrm{~d}$, $J=7.7 \mathrm{~Hz}, 1 \mathrm{H}), 7.52(\mathrm{td}, J=7.5 \mathrm{~Hz}, J=1.4 \mathrm{~Hz}, 1 \mathrm{H}), 7.42-7.26(\mathrm{~m}, 6 \mathrm{H}), 7.01(\mathrm{~d}, J=8.8 \mathrm{~Hz}$, $2 \mathrm{H}), 6.75(\mathrm{~d}, J=8.8 \mathrm{~Hz}, 2 \mathrm{H}), 6.72(\mathrm{~d}, J=7.7 \mathrm{~Hz}, 1 \mathrm{H}), 6.66(\mathrm{~s}, 1 \mathrm{H}), 6.63(\mathrm{~d}, J=2.2 \mathrm{~Hz}, 1 \mathrm{H})$, 
$5.93(\mathrm{~d}, J=4.9 \mathrm{~Hz}, 2 \mathrm{H}), 5.33(\mathrm{~d}, J=5.9 \mathrm{~Hz}, 1 \mathrm{H}), 4.97(\mathrm{~s}, 2 \mathrm{H}), 4.61(\mathrm{~d}, J=5.9 \mathrm{~Hz}, 1 \mathrm{H}) ;{ }^{13} \mathrm{C}$ NMR (75 MHz, CD 3 OD): d (ppm) 174.2, 165.3, 158.4, 147.6, 146.5, 137.6, 137.3, 135.6, 135.5, 134.6, 132.0, 129.9, 129.4 (2C), 128.8, 128.6, 128.0, 127.3, 127.2, 127.1, 126.4, 120.5, $113.9(2 \mathrm{C})$, 108.5, 107.4, 101.4, 69.5, 66.5, 52.8; HRMS (E.S.I. ${ }^{+}, \mathrm{m} / \mathrm{z}$ ) calcd for $\mathrm{C}_{30} \mathrm{H}_{24} \mathrm{NO}_{6}{ }^{+}(\mathrm{M}+\mathrm{H})^{+}$: 494.1598, found: 494.1574 .

2-(4-methoxybenzyl)-3-(4-methoxyphenyl)-1-oxo-1,2,3,4-tetrahydroisoquinoline-4-carboxylic acid $(2 d)$ : The title compound was prepared following the general procedure described above using 1d (25.5 mg, $0.100 \mathrm{mmol}, 1.0$ eq.) and IVa (24.3 mg, $0.150 \mathrm{mmol}, 1.5$ eq.) in $20 \mathrm{~min}$ of reaction time. The product $\mathbf{2 d}$ was isolated in $63 \%$ yield $(26.3 \mathrm{mg}$, d.r. (cis / trans): $>19 / 1$ ) as a light amorphous solid. When the reaction was performed at room temperature in 2 min of reaction time: $40.5 \mathrm{mg}, 97 \%$, d.r. (cis/trans): 2.2/1 with separation. Cis product (2d): light amorphous solid (27.8 mg, 67\%). Trans product (2d'): light amorphous solid (12.5 mg, 30\%). Cis product 2d: IR (v): 2928, 1726, 1613, 1572, 1511, 1470, 1249, 1176, 1031, 832, $735 \mathrm{~cm}^{-1} ;{ }^{1} \mathrm{H}$ NMR (300 MHz, CD $\left.3 \mathrm{OD}\right): \mathrm{d}(\mathrm{ppm}) 8.00(\mathrm{dd}, J=7.8 \mathrm{~Hz}, J=1.5 \mathrm{~Hz}, 1 \mathrm{H})$, $7.58(\mathrm{~d}, J=7.8 \mathrm{~Hz}, 1 \mathrm{H}), 7.41-7.20(\mathrm{~m}, 3 \mathrm{H}), 7.11(\mathrm{~d}, J=8.7 \mathrm{~Hz}, 2 \mathrm{H}), 6.86(\mathrm{~d}, J=8.7 \mathrm{~Hz}, 2 \mathrm{H})$, $6.77(\mathrm{~d}, J=8.7 \mathrm{~Hz}, 2 \mathrm{H}), 6.59(\mathrm{~d}, J=8.7 \mathrm{~Hz}, 2 \mathrm{H}), 5.31(\mathrm{~d}, J=14.5 \mathrm{~Hz}, 1 \mathrm{H}), 4.88(\mathrm{~d}, J=6.4 \mathrm{~Hz}$, 1H) $4.21(\mathrm{~d}, J=6.4 \mathrm{~Hz}, 1 \mathrm{H}), 3.67(\mathrm{~s}, 3 \mathrm{H}), 3.60(\mathrm{~s}, 3 \mathrm{H}), 3.56(\mathrm{~d}, J=14.5 \mathrm{~Hz}, 1 \mathrm{H}) ;{ }^{13} \mathrm{C} \mathrm{NMR}$ (75 MHz, CD 3 OD): d (ppm) 166.6, 160.9, 160.7, 138.2, 136.1, 133.3, 131.8, 130.6 (2C), 130.5 (2C), 130.2, 129.9, 128.8, 128.4, 127.8, 115.1 (2C), 114.6 (2C), 62.5, 55.7, 55.6, 53.5, 48.9; HRMS (E.S.I. ${ }^{+}, \mathrm{m} / \mathrm{z}$ ) calcd for $\mathrm{C}_{25} \mathrm{H}_{24} \mathrm{NO}_{5}{ }^{+}(\mathrm{M}+\mathrm{H})^{+}:$418.1649, found: 418.1667. Trans product 2d': IR (v): 2960, 2926, 1728, 1611, 1574, 1510, 1466, 1246, 1174, 1029, 799, $733 \mathrm{~cm}^{-1} ;{ }^{1} \mathrm{H}$ $\operatorname{NMR}\left(300 \mathrm{MHz}, \mathrm{CDCl}_{3}\right): \mathrm{d}(\mathrm{ppm}) 8.16(\mathrm{dd}, J=6.9 \mathrm{~Hz}, J=2.2 \mathrm{~Hz}, 1 \mathrm{H}), 7.41-7.31(\mathrm{~m}, 3 \mathrm{H})$, $7.10(\mathrm{~d}, J=8.6 \mathrm{~Hz}, 2 \mathrm{H}), 7.01(\mathrm{dd}, J=6.5 \mathrm{~Hz}, J=1.8 \mathrm{~Hz}, 1 \mathrm{H}), 6.87(\mathrm{~d}, J=8.6 \mathrm{~Hz}, 2 \mathrm{H}), 6.67(\mathrm{~d}$, $J=8.7 \mathrm{~Hz}, 2 \mathrm{H}), 6.65(\mathrm{~d}, J=8.7 \mathrm{~Hz}, 2 \mathrm{H}), 5.50(\mathrm{~d}, J=14.5 \mathrm{~Hz}, 1 \mathrm{H}), 4.98(\mathrm{~s}, 1 \mathrm{H}), 3.75(\mathrm{~s}, 1 \mathrm{H})$, $3.66(\mathrm{~s}, 3 \mathrm{H}), 3.63(\mathrm{~s}, 3 \mathrm{H}), 3.56(\mathrm{~d}, J=14.5 \mathrm{~Hz}, 1 \mathrm{H}) ;{ }^{13} \mathrm{C} \mathrm{NMR}\left(75 \mathrm{MHz}, \mathrm{CDCl}_{3}\right)$ : d (ppm) 175.0, 163.7, 159.3, 159.1, 132.1, 131.5, 130.2 (2C), 129.4, 129.1, 128.8, 128.6, 128.3, 127.5 (2C), 114.2 (2C), 113.7 (2C), 59.5, 55.2, 55.1, 51.1, 48.2; HRMS (E.S.I. ${ }^{+}, \mathrm{m} / \mathrm{z}$ ) calcd for $\mathrm{C}_{25} \mathrm{H}_{24} \mathrm{NO}_{5}{ }^{+}$ $(\mathrm{M}+\mathrm{H})^{+}: 418.1649$, found: 418.1609 .

Cis-2-(tert-butyl)-3-(4-methoxyphenyl)-1-oxo-1,2,3,4-tetrahydroisoquinoline-4-carboxylic acid (2e): The title compound was prepared following the general procedure described above using the brut 1e (0.100 mmol, 1.0 eq.) and IVa (24.3 mg, $0.150 \mathrm{mmol}, 1.5$ eq.) in $40 \mathrm{~min}$ of reaction time. The product $\mathbf{2 e}$ was isolated in $69 \%$ yield $(24.4 \mathrm{mg}$, d.r. (cis/trans): $>19 / 1)$ as a white powder. When the reaction was performed at room temperature in 2 min of reaction time: $27.9 \mathrm{mg}, 79 \%$, d.r. (cis/trans): $>19 / 1$. Mp: $60-62{ }^{\circ} \mathrm{C}$; IR (v): 2923, 2510, 1716, 1653, 1603, 1511, 1390, 1254, 1161, 1025, 788, $756 \mathrm{~cm}^{-1},{ }^{1} \mathrm{H}$ NMR (500 MHz, CD OD): d (ppm) $8.03(\mathrm{~d}, J=7.7 \mathrm{~Hz}, 1 \mathrm{H}), 7.72(\mathrm{~d}, J=7.7 \mathrm{~Hz}, 1 \mathrm{H}), 7.41(\mathrm{dd}, J=7.7 \mathrm{~Hz}, J=7.7 \mathrm{~Hz}, 1 \mathrm{H})$, $7.34(\mathrm{dd}, J=7.7 \mathrm{~Hz}, J=7.7 \mathrm{~Hz}, 1 \mathrm{H}), 6.99(\mathrm{~d}, J=8.6 \mathrm{~Hz}, 2 \mathrm{H}), 6.67(\mathrm{~d}, J=8.6 \mathrm{~Hz}, 2 \mathrm{H}), 5.58$ $(\mathrm{d}, J=5.6 \mathrm{~Hz}, 1 \mathrm{H}), 4.43(\mathrm{~d}, J=5.6 \mathrm{~Hz}, 1 \mathrm{H}), 3.70(\mathrm{~s}, 3 \mathrm{H}), 1.52(\mathrm{~s}, 9 \mathrm{H}) ;{ }^{13} \mathrm{C} \mathrm{NMR}(125 \mathrm{MHz}$, $\left.\mathrm{CDCl}_{3}\right)$ : d (ppm) 170.8, 162.3, 144.4, 132.7, 132.0, 130.6 (2C), 130.1, 129.7, 128.0, 127.7, 114.7, 114.3 (2C), 60.7, 60.3, 55.6, 54.6, 29.2 (3C); HRMS (E.S.I. ${ }^{+}, \mathrm{m} / \mathrm{z}$ ) calcd for $\mathrm{C}_{21} \mathrm{H}_{24} \mathrm{NO}_{4}{ }^{+}(\mathrm{M}+$ $\mathrm{H})^{+}: 354.1700$, found: 354.1745 .

Cis-3-isopropyl-1-oxo-2-phenyl-1,2,3,4-tetra hydroisoquinoline-4-carboxylic acid (2f): The title compound was prepared following the general procedure described above using the brut 1f (0.100 mmol, 1.0 eq.) and IVa (24.3 mg, $0.150 \mathrm{mmol}, 1.5$ eq.) in $40 \mathrm{~min}$ of reaction time. The product $\mathbf{2} \mathbf{f}$ was isolated in $41 \%$ yield $(12.8 \mathrm{mg}$, d.r. (cis/trans): $>19 / 1)$ as a light amorphous solid. When the reaction was performed at room temperature in 5 min of reaction time: $12.4 \mathrm{mg}, 40 \%$, d.r. (cis/trans): $>19 / 1$. IR (v): 3398, 2963, 1710, 1599, 1580, 1552, 1497, 1381, 1261, 1027, 799, $755 \mathrm{~cm}^{-1} ;{ }^{1} \mathrm{H}$ NMR (300 MHz, CD 3 OD): d (ppm) 7.96 $(\mathrm{dd}, J=7.5 \mathrm{~Hz}, J=1.5 \mathrm{~Hz}, 2 \mathrm{H}), 7.58(\mathrm{dd}, J=8.1 \mathrm{~Hz}, J=1.5 \mathrm{~Hz}, 2 \mathrm{H}), 7.52(\mathrm{dd}, J=7.5 \mathrm{~Hz}$, $J=1.3 \mathrm{~Hz}, 1 \mathrm{H}), 7.5(\mathrm{t}, J=7.5 \mathrm{~Hz}, 2 \mathrm{H}), 7.37(\mathrm{dd}, J=8.1 \mathrm{~Hz}, J=1.3 \mathrm{~Hz}, 1 \mathrm{H}), 7.30(\mathrm{~d}, J=7.5 \mathrm{~Hz}$, $1 \mathrm{H}), 4.55(\mathrm{dd}, J=5.6 \mathrm{~Hz}, J=3.3 \mathrm{~Hz}, 1 \mathrm{H}), 4.50(\mathrm{~d}, J=5.6 \mathrm{~Hz}, 1 \mathrm{H}), 3.66(\mathrm{~b}-\mathrm{s}, 1 \mathrm{H}, \mathrm{OH}), 2.27$ $(\mathrm{ddd}, J=7.1 \mathrm{~Hz}, J=7.1 \mathrm{~Hz}, J=3.3 \mathrm{~Hz}, 1 \mathrm{H}), 0.73(\mathrm{~d}, J=7.1 \mathrm{~Hz}, 3 \mathrm{H}), 0.68(\mathrm{~d}, J=7.1 \mathrm{~Hz}, 3 \mathrm{H})$; ${ }^{13} \mathrm{C}$ NMR (125 MHz, CD $\left.\mathrm{OD}\right)$ : d (ppm) 176.6, 166.6, 144.8, 140.0, 133.2, 130.9, 129.7 (2C), 
129.2, 128.8 (2C), 128.5, 127.7, 127.6, 68.6, 52.5, 32.5, 23.0, 19.8; HRMS (E.S.I. ${ }^{+}, \mathrm{m} / \mathrm{z}$ ) calcd for $\mathrm{C}_{19} \mathrm{H}_{20} \mathrm{NO}_{3}{ }^{+}(\mathrm{M}+\mathrm{H})^{+}: 310.1438$, found: 310.1452 .

Cis-2-cyclopropyl-1-oxo-3-(thiophen-2-yl)-1,2,3,4-tetrahydroisoquinoline-4-carboxylic acid $(2 \mathrm{~g})$ : A round-bottom flask under an argon atmosphere was charged with the brut $\mathbf{1 g}$ $(0.100 \mathrm{mmol}, 1.0$ eq. $)$ in TFE $(3 \mathrm{~mL})$ and then charged with homophthalic anhydride IVa $(24.3 \mathrm{mg}, 0.150 \mathrm{mmol}, 1.5 \mathrm{eq}$.). The reaction mixture was stirred for $8 \mathrm{~min}$ at room temperature. The mixture was concentrated in vacuo, and the residue was purified by preparative TLC to afford the pure products $\mathbf{2 g}$ and $\mathbf{2} \mathbf{g}^{\prime}$. Purification: $\mathrm{CH}_{2} \mathrm{Cl}_{2} / \mathrm{MeOH}$ (93:7). The product $2 \mathrm{~g}$ (cis product) was isolated in $58 \%$ yield (18.2 $\mathrm{mg}$, white powder). The product $\mathbf{2} \mathbf{g}^{\prime}$ (trans product) was isolated in $32 \%$ yield $(10.1 \mathrm{mg}$, white powder). Cis product 2g: Mp: $228-229{ }^{\circ} \mathrm{C}$; IR ( v): 3428, 3075, 3011, 1714, 1642, 1598, 1462, 1423, 1359, 1301, 1244, 1226, 1027, 907, 827, $697 \mathrm{~cm}^{-1} ;{ }^{1} \mathrm{H}$ NMR (500 MHz, CD $\mathrm{CD}_{3}$ ) d (ppm) 8.09 (d, $J=7.6 \mathrm{~Hz}, 1 \mathrm{H}), 7.88(\mathrm{~d}, J=7.6 \mathrm{~Hz}, 1 \mathrm{H}), 7.55(\mathrm{t}, J=7.6 \mathrm{~Hz}, 1 \mathrm{H}), 7.43(\mathrm{t}, J=7.6 \mathrm{~Hz}, 1 \mathrm{H}), 7.14$ $(\mathrm{d}, J=4.7 \mathrm{~Hz}, 1 \mathrm{H}), 6.89(\mathrm{~d}, J=2.7 \mathrm{~Hz}, 1 \mathrm{H}), 6.83(\mathrm{dd}, J=4.7 \mathrm{~Hz}, J=2.7 \mathrm{~Hz}, 1 \mathrm{H}), 5.50(\mathrm{~d}$, $J=5.7 \mathrm{~Hz}, 1 \mathrm{H}), 4.55(\mathrm{~d}, J=5.7 \mathrm{~Hz}, 1 \mathrm{H}), 2.68-2.63(\mathrm{~m}, 1 \mathrm{H}), 1.06-1.01(\mathrm{~m}, 1 \mathrm{H}), 0.91-0.85(\mathrm{~m}$, 2H), 0.85-0.80 (m, 1H); ${ }^{13} \mathrm{C}$ NMR (125 MHz, CD $\left.\mathrm{OD}\right): \mathrm{d}$ (ppm) 173.6, 168.2, 141.6, 136.7, 133.6, 130.2, 130.0, 128.7, 128.4, 128.3, 126.9, 126.4, 61.6, 51.7, 30.8, 9.7, 6.4; HRMS (E.S.I. ${ }^{+}$, $\mathrm{m} / \mathrm{z}$ ) calcd for $\mathrm{C}_{17} \mathrm{H}_{16} \mathrm{NO}_{3} \mathrm{~S}^{+}(\mathrm{M}+\mathrm{H})^{+}:$314.0845, found: 314.0853. Trans product $2 \mathrm{~g}^{\prime}: \mathrm{Mp}$ : 238-239 ${ }^{\circ} \mathrm{C}$; IR (v): 3395, 3076, 3011, 1709, 1638, 1598, 1580, 1465, 1432, 1359, 1245, 1156, 1028, 966, 826, 728, $698 \mathrm{~cm}^{-1} ;{ }^{1} \mathrm{H}$ NMR (500 MHz, CD $\left.3 \mathrm{OD}\right): \mathrm{d}(\mathrm{ppm}) 8.00$ (d, J = 7.6 Hz, $1 \mathrm{H}), 7.47(\mathrm{t}, J=7.6 \mathrm{~Hz}, 1 \mathrm{H}), 7.37(\mathrm{t}, J=7.6 \mathrm{~Hz}, 1 \mathrm{H}), 7.29(\mathrm{~d}, J=7.6 \mathrm{~Hz}, 1 \mathrm{H}), 7.15(\mathrm{~d}, J=4.8$ $\mathrm{Hz}, 1 \mathrm{H}), 6.87(\mathrm{~d}, J=2.8 \mathrm{~Hz}, 1 \mathrm{H}), 6.85(\mathrm{dd}, J=4.8 \mathrm{~Hz}, J=2.8 \mathrm{~Hz}, 1 \mathrm{H}), 5.68(\mathrm{~s}, 1 \mathrm{H}), 3.93(\mathrm{~s}$, $1 \mathrm{H}), 2.83-2.78(\mathrm{~m}, 1 \mathrm{H}), 1.05-0.97(\mathrm{~m}, 1 \mathrm{H}), 0.88-0.82(\mathrm{~m}, 2 \mathrm{H}), 0.82-0.76(\mathrm{~m}, 1 \mathrm{H}) ;{ }^{13} \mathrm{C} \mathrm{NMR}$ (125 MHz, CD 3 OD): d (ppm) 176.0, 167.9, 145.8, 138.2, 133.4, 131.1, 130.0, 128.3 (2C), 127.4, 126.1, 125.5, 62.2, 55.5, 31.0, 9.6, 6.5; HRMS (E.S.I. ${ }^{+}, \mathrm{m} / \mathrm{z}$ ) calcd for $\mathrm{C}_{17} \mathrm{H}_{16} \mathrm{NO}_{3} \mathrm{~S}^{+}(\mathrm{M}+\mathrm{H})^{+}$: 314.0845, found: 314.0839 .

Trans-2-butyl-3-(4-nitrophenyl)-1-oxo-1,2,3,4-tetrahydroisoquinoline-4-carboxylic acid (2h): A round-bottom flask under an argon atmosphere was charged with the brut 1 h $(0.100 \mathrm{mmol}$, 1.0 eq.) in TFE ( $3 \mathrm{~mL})$ and then charged with homophthalic anhydride IVa $(24.3 \mathrm{mg}$, $0.150 \mathrm{mmol}, 1.5$ eq.). The reaction mixture was stirred for $2 \mathrm{~min}$ at room temperature. The mixture was concentrated in vacuo, and residue was purified by preparative TLC to afford the pure products $\mathbf{2} \mathbf{h}$ and $\mathbf{2} \mathbf{h}^{\prime}$. Purification: $\mathrm{CH}_{2} \mathrm{Cl}_{2} / \mathrm{MeOH}$ (93:7). The product $\mathbf{2 h}$ (trans product) was isolated in $48 \%$ yield $\left(17.5 \mathrm{mg}\right.$, white powder). The product $\mathbf{2} \mathbf{h}^{\prime}$ (cis product) was isolated in $29 \%$ yield (10.8 mg, white powder). Trans product $2 \mathrm{~h}: \mathrm{Mp}: 223-224{ }^{\circ} \mathrm{C}$; IR (v): 3415, 2959, 2932, 2871, 1708, 1636, 1599, 1518, 1472, 1344, 1263, 1164, 1109, 908, 853, 712 $\mathrm{cm}^{-1}{ }^{1} \mathrm{H}$ NMR (300 MHz, CD $\left.\mathrm{CD}_{3} \mathrm{OD}\right): \mathrm{d}(\mathrm{ppm}) 8.09$ (d, $\left.J=8.8 \mathrm{~Hz}, 2 \mathrm{H}\right), 8.00(\mathrm{dd}, J=7.1 \mathrm{~Hz}$, $J=2.1 \mathrm{~Hz}, 1 \mathrm{H}), 7.42-7.32(\mathrm{~m}, 2 \mathrm{H}), 7.38(\mathrm{~d}, J=8.8 \mathrm{~Hz}, 2 \mathrm{H}), 7.11(\mathrm{dd}, J=6.5 \mathrm{~Hz}, J=2.1 \mathrm{~Hz}$, $1 \mathrm{H}), 5.59(\mathrm{~d}, J=1.0 \mathrm{~Hz}, 1 \mathrm{H}), 4.04(\mathrm{dt}, J=13.3 \mathrm{~Hz}, J=8.0 \mathrm{~Hz}, 1 \mathrm{H}), 3.86(\mathrm{~d}, J=1.0 \mathrm{~Hz}, 1 \mathrm{H})$, $2.96(\mathrm{ddd}, J=13.3 \mathrm{~Hz}, J=8.0 \mathrm{~Hz}, J=6.5 \mathrm{~Hz}, 1 \mathrm{H}), 1.72-1.61(\mathrm{~m}, 2 \mathrm{H}), 1.41-1.33(\mathrm{~m}, 2 \mathrm{H})$, $0.94(\mathrm{t}, J=7.3 \mathrm{~Hz}, 3 \mathrm{H}) ;{ }^{13} \mathrm{C} \mathrm{NMR}\left(75 \mathrm{MHz}, \mathrm{CD}_{3} \mathrm{OD}\right): \mathrm{d}(\mathrm{ppm})$ 174.4, 165.0, 148.0, 147.2, 135.5, 131.7, 129.1, 128.6, 127.0 (3C), 126.6, 123.1 (2C), 62.5, 53.8, 46.6, 29.4, 19.8, 12.6; HRMS (E.S.I. ${ }^{+}, \mathrm{m} / \mathrm{z}$ ) calcd for $\mathrm{C}_{20} \mathrm{H}_{21} \mathrm{~N}_{2} \mathrm{O}_{5}{ }^{+}(\mathrm{M}+\mathrm{H})^{+}: 369.1445$, found: 369.1440. Cis product 2h': Mp: $232-233{ }^{\circ} \mathrm{C}$; IR (v): 3386, 2958, 2931, 2872, 1727, 1633, 1598, 1519, 1471, 1377, 1345, 1313, $1255,1163,1110,1014,909,855,799,729,715 \mathrm{~cm}^{-1} ;{ }^{1} \mathrm{H}$ NMR (500 MHz, CD 3 OD): d (ppm) $8.08(\mathrm{~d}, J=7.4 \mathrm{~Hz}, 1 \mathrm{H}), 8.03(\mathrm{~d}, J=8.3 \mathrm{~Hz}, 2 \mathrm{H}), 7.72(\mathrm{~d}, J=7.4 \mathrm{~Hz}, 1 \mathrm{H}), 7.49(\mathrm{dd}, J=7.4 \mathrm{~Hz}$, $J=7.4 \mathrm{~Hz}, 1 \mathrm{H}), 7.41(\mathrm{dd}, J=7.4 \mathrm{~Hz}, J=7.4 \mathrm{~Hz}, 1 \mathrm{H}), 7.32(\mathrm{~d}, J=8.3 \mathrm{~Hz}, 2 \mathrm{H}), 5.29(\mathrm{~d}, J=6.2 \mathrm{~Hz}$, $1 \mathrm{H}), 4.55(\mathrm{~d}, J=6.2 \mathrm{~Hz}, 1 \mathrm{H}), 4.02(\mathrm{ddd}, J=14.4 \mathrm{~Hz}, J=8.0 \mathrm{~Hz}, J=8.0 \mathrm{~Hz}, 1 \mathrm{H}), 2.91(\mathrm{ddd}$, $J=14.4 \mathrm{~Hz}, J=8.0 \mathrm{~Hz}, J=5.5 \mathrm{~Hz}, 1 \mathrm{H}), 1.71-1.59(\mathrm{~m}, 2 \mathrm{H}), 1.44-1.35(\mathrm{~m}, 2 \mathrm{H}), 0.95(\mathrm{t}, J=7.3 \mathrm{~Hz}$, $3 \mathrm{H}) ;{ }^{13} \mathrm{C}$ NMR (125 MHz, CD $\left.\mathrm{OD}\right): \mathrm{d}$ (ppm) 175.5, 166.4, 149.0, 146.9, 137.3, 133.4, 130.5 (2C), 130.1, 130.0, 128.4, 128.1, 124.0 (2C), 63.6, 53.6, 47.6, 31.2, 21.2, 14.1; HRMS (E.S.I. ${ }^{+}$, $\mathrm{m} / \mathrm{z}) \mathrm{C}_{20} \mathrm{H}_{21} \mathrm{~N}_{2} \mathrm{O}_{5}{ }^{+}(\mathrm{M}+\mathrm{H})^{+}: 369.1445$, found: 369.1447 .

Cis-3-(4-methoxyphenyl)-1-oxo-2-(4-(trifluoromethyl)phenyl)-1,2,3,4-tetrahydroisoquinoline4-carboxylic acid (2i): A round-bottom flask under an argon atmosphere was charged with the brut $1 \mathrm{i}(0.100 \mathrm{mmol}, 1.0$ eq.) in TFE $(3 \mathrm{~mL})$ and then charged with homophthalic 
anhydride IVa (24.3 mg, $0.150 \mathrm{mmol}, 1.5$ eq.). The reaction mixture was stirred for $8 \mathrm{~min}$ at room temperature. The mixture was concentrated in vacuo, and the residue was purified by preparative TLC to afford the pure product 2 i. Purification: $\mathrm{CH}_{2} \mathrm{Cl}_{2} / \mathrm{MeOH}$ (93:7). The product $2 \mathbf{i}$ was isolated in $80 \%$ yield $(28.3 \mathrm{mg}$, d.r. (cis/trans): $5 / 1$ ) as a white powder. Mp: $238-239{ }^{\circ} \mathrm{C}$; IR (v): 3427, 3075, 2935, 2839, 1719, 1648, 1603, 1513, 1460, 1391, 1322, $1249,1162,1115,1060,1018,930,734,693 \mathrm{~cm}^{-1} ;{ }^{1} \mathrm{H}$ NMR (300 MHz, CD 3 OD): d (ppm) 8.13 $(\mathrm{dd}, J=7.7 \mathrm{~Hz}, J=1.1 \mathrm{~Hz}, 1 \mathrm{H}), 7.75(\mathrm{~d}, J=7.7 \mathrm{~Hz}, 1 \mathrm{H}), 7.60(\mathrm{~d}, J=8.3 \mathrm{~Hz}, 2 \mathrm{H}), 7.57(\mathrm{ddd}$, $J=7.7 \mathrm{~Hz}, J=7.7 \mathrm{~Hz}, J=1.1 \mathrm{~Hz}, 1 \mathrm{H}), 7.45(\mathrm{dd}, J=7.7 \mathrm{~Hz}, J=7.7 \mathrm{~Hz}, 1 \mathrm{H}), 7.42(\mathrm{~d}$, $J=8.3 \mathrm{~Hz}, 2 \mathrm{H}), 7.05(\mathrm{~d}, J=8.8 \mathrm{~Hz}, 2 \mathrm{H}), 6.69(\mathrm{~d}, J=8.8 \mathrm{~Hz}, 2 \mathrm{H}), 5.52(\mathrm{~d}, J=5.6 \mathrm{~Hz}, 2 \mathrm{H}), 4.64(\mathrm{~d}$, $J=5.6 \mathrm{~Hz}, 1 \mathrm{H}), 3.69$ (s, 3H); ${ }^{13} \mathrm{C}$ NMR $\left(75 \mathrm{MHz}, \mathrm{CD}_{3} \mathrm{OD}\right): \mathrm{d}(\mathrm{ppm}) 174.6,160.9,146.6,138.3$, 136.2, 133.8, 132.4, 131.5, 130.8 (2C), 130.4, 130.0, 129.6 (2C), 129.0, 128.3, 126.8, 126.7, 114.6 (2C), 66.8, 55.6, 53.4; ${ }^{19} \mathrm{~F}$ NMR $\left(280 \mathrm{MHz}, \mathrm{CD}_{3} \mathrm{OD}\right)$ : d (ppm) -63.96 (s, $\left.\mathrm{CF}_{3}\right)$; HRMS (E.S.I. ${ }^{+}$, $\mathrm{m} / \mathrm{z}$ ) calcd for $\mathrm{C}_{24} \mathrm{H}_{19} \mathrm{~F}_{3} \mathrm{NO}_{4}{ }^{+}(\mathrm{M}+\mathrm{H})^{+}:$442.1261, found: 442.1253 .

Trans-3-(ethoxycarbonyl)-2-(4-methoxyphenyl)-1-oxo-1,2,3,4-tetrahydroisoquinoline-4-carboxylic acid $(\mathbf{2} \mathbf{j})$ : A round-bottom flask under an argon atmosphere was charged with the brut $\mathbf{1 j}$ ( $0.100 \mathrm{mmol}, 1.0$ eq.) in TFE $(3 \mathrm{~mL})$ and then charged with homophthalic anhydride IVa ( $24.3 \mathrm{mg}, 0.150 \mathrm{mmol}, 1.5 \mathrm{eq}$.). The reaction mixture was stirred $8 \mathrm{~min}$ at room temperature. The mixture was concentrated in vacuo, and residue was purified by preparative TLC to afford the pure product $\mathbf{2} \mathbf{j}$. Purification: $\mathrm{CH}_{2} \mathrm{Cl}_{2} / \mathrm{MeOH}(93: 7)$. The product $\mathbf{2} \mathbf{j}$ was isolated in $68 \%$ yield $(25.0 \mathrm{mg}$, d.r. (trans / cis): $>19 / 1)$ as a brown powder. Mp: $224-225{ }^{\circ} \mathrm{C}$; IR (v): 3386, 2960, 2936, 1738, 1634, 1599, 1509, 1462, 1429, 1364, 1299, 1241, 1178, 1026, 912, 834, 711 $\mathrm{cm}^{-1}{ }^{1} \mathrm{H}$ NMR (300 MHz, CD $\left.\mathrm{OD}\right): \mathrm{d}(\mathrm{ppm}) 8.00(\mathrm{dd}, J=7.5 \mathrm{~Hz}, J=1.2 \mathrm{~Hz}, 1 \mathrm{H}), 7.75$ (ddd, $J=7.5 \mathrm{~Hz}, J=7.5 \mathrm{~Hz}, J=1.4 \mathrm{~Hz}, 1 \mathrm{H}), 7.44(\mathrm{~d}, J=8.9 \mathrm{~Hz}, 2 \mathrm{H}), 7.46-7.40(\mathrm{~m}, 2 \mathrm{H}), 6.98(\mathrm{~d}$, $J=8.9 \mathrm{~Hz}, 2 \mathrm{H}), 5.20(\mathrm{~d}, J=1.7 \mathrm{~Hz}, 1 \mathrm{H}), 4.24(\mathrm{~d}, J=1.7 \mathrm{~Hz}, 1 \mathrm{H}), 4.08(\mathrm{q}, J=7.1 \mathrm{~Hz}, 2 \mathrm{H}), 3.83$ $(\mathrm{s}, 3 \mathrm{H}), 1.11(\mathrm{t}, J=7.1 \mathrm{~Hz}, 3 \mathrm{H}) ;{ }^{13} \mathrm{C}$ NMR $\left(175 \mathrm{MHz}, \mathrm{CD}_{3} \mathrm{OD}\right): \mathrm{d}(\mathrm{ppm}) 175.8,172.7,166.6$, 160.2, 138.6, 136.6, 133.2, 130.5, 129.9, 129.7 (2C), 128.6, 128.2, 115.1 (2C), 67.8, 62.7, 55.9, 51.7, 14.3; HRMS (E.S.I. ${ }^{+}, \mathrm{m} / \mathrm{z}$ ) calcd for $\mathrm{C}_{20} \mathrm{H}_{20} \mathrm{NO}_{6}{ }^{+}(\mathrm{M}+\mathrm{H})^{+}: 370.1285$, found: 370.1283 .

\section{Conclusions}

In summary, an efficient, simple, and rapid cycloaddition of homophthalic anhydride with imines has been described to enable access to a variety of densely substituted 3,4-lactams. This reaction typically proceeds with high diastereoselectivity for cis-kinetic or trans-thermodynamic diastereoisomers, depending on the substituent's properties attached to the starting imine's carbon. We demonstrated for the first time that TFE could act simultaneously as a solvent and as a powerful catalyst for CCR without generating by-products.

Supplementary Materials: The following are available online, copies of $1 \mathrm{H}$ and $13 \mathrm{C}$ spectra for all prepared.

Author Contributions: Conceptualization, T.B. and C.G.; writing the experimental part T.B.; writingreview and editing, C.G.; supervision, C.G.; project administration, C.G.; funding acquisition, T.B. All authors have read and agreed to the published version of the manuscript.

Funding: This research was funded by the labex Laboratoire d'Excellence en Recherche sur le Médicament et l'Innovation Thérapeutique LERMIT- Université de Paris-Saclay.

Institutional Review Board Statement: Not applicable.

Informed Consent Statement: Not applicable.

Data Availability Statement: Not applicable.

Acknowledgments: We thank ICSN, CNRS, and Labex Lermit for their financial supports. T.B. thanks Labex Lermit for a doctoral fellowship, Université Paris-Saclay.

Conflicts of Interest: The funders had no role in the design of the study; in the collection, analyses, or interpretation of data; in the writing of the manuscript, or in the decision to publish the results. 


\section{References}

1. Castagnoli, N. Condensation of succinic anhydride with N-benzylidene-N-methylamine. Stereoselective synthesis of trans- and cis-1-methyl-4-carboxy-5-phenyl-2-pyrrolidinone. J. Org. Chem. 1969, 34, 3187-3189. [CrossRef] [PubMed]

2. Castagnoli, N.; Cushman, M. Condensation of succinic anhydrides with Schiff bases. Scope and mechanism. J. Org. Chem. 1971, 36, 3404-3406. [CrossRef] [PubMed]

3. Gonzalez-Lopez, M.; Shaw, J.T. Cyclic Anhydrides in Formal Cycloadditions and Multicomponent Reactions. Chem. Rev. 2009, 109, 164-189. [CrossRef] [PubMed]

4. Weintraub, P.; Sabol, J.; Kane, J.; Borcherding, D. Recent advances in the synthesis of piperidones and piperidines. Tetrahedron 2003, 59, 2953-2989. [CrossRef]

5. Di Maso, M.J.; Snyder, K.M.; De Souza Fernandes, F.; Pattawong, O.; Tan, D.Q.; Fettinger, J.C.; Cheong, P.H.Y.; Shaw, J.T. Diastereoselective Synthesis of and Mechanistic Understanding for the Formation of 2-Piperidinones from Imines and CyanoSubstituted Anhydrides. Chem. Eur. J. 2016, 22, 4794-4801. [CrossRef]

6. Siu, T.; Altman, M.D.; Baltus, G.A.; Childers, M.; Ellis, J.M.; Gunaydin, H.; Hatch, H.; Ho, T.; Jewell, J.; Lacey, B.M.; et al. Discovery of a Novel cGAMP Competitive Ligand of the Inactive Form of STING. ACS Med. Chem. Lett. 2019, 10, 92-97. [CrossRef]

7. Floyd, D.M.; Stein, P.; Wang, Z.; Liu, J.; Castro, S.; Clark, J.A.; Connelly, M.; Zhu, F.; Holbrook, G.; Matheny, A.; et al. Hit-to-Lead Studies for the Antimalarial Tetrahydroisoquinolone Carboxanilides. J. Med. Chem. 2016, 59, 7950-7962. [CrossRef]

8. Cinelli, M.A.; Reddy, P.V.N.; Lv, P.C.; Liang, J.H.; Chen, L.; Agama, K.; Pommier, Y.; van Breemen, R.B.; Cushman, M. Identification, Synthesis, and Biological Evaluation of Metabolites of the Experimental Cancer Treatment Drugs Indotecan (LMP400) and Indimitecan (LMP776) and Investigation of Isomerically Hydroxylated Indenoisoquinoline Analogues as Topoisomerase I Poisons. J. Med. Chem. 2012, 55, 10844-10862. [CrossRef]

9. Nguyen, T.X.; Abdelmalak, M.; Marchand, C.; Agama, K.; Pommier, Y.; Cushman, M. Synthesis and biological evaluation of nitrated 7-, 8-, 9-, and 10-hydroxyindenoisoquinolines as potential dual topoisomerase I (Top1)-tyrosyl-DNA phosphodiesterase I (TDP1) inhibitors. J. Med. Chem. 2015, 58, 3188-3208. [CrossRef]

10. Chatterjee, M.; Hartung, A.; Holzgrabe, U.; Mueller, E.; Peinz, U.; Sotriffer, C.; Zilian, D. WO 2015185114A1. 2015. Available online: https: / / worldwide.espacenet.com/publicationDetails/biblio?II=0\&ND=3\&adjacent=true\&locale=fr_EP\&FT=D\&date= 20151210\&CC $=$ WO\&NR=2015185114A1\&KC=A1 (accessed on 23 December 2021).

11. Cushman, M.; Gentry, J.; Dekow, F.W. Condensation of imines with homophthalic anhydrides. A convergent synthesis of cis- and trans-13-methyltetrahydroprotoberberines. J. Org. Chem. 1977, 42, 1111-1116. [CrossRef]

12. Iwasa, K.; Gupta, Y.P.; Cushman, M. Absolute configurations of the cis- and trans-13-methyltetrahydroprotoberberines. Total synthesis of $(+)$-thalictricavine, $(+)$-canadine, $( \pm)-,(-)-$, and (+)-thalictrifoline, and $( \pm),(-)-$, and (+)-cavidine. J. Org. Chem. 1981, 46, 4744-4750. [CrossRef]

13. Iwasa, K.; Gupta, Y.P.; Cushman, M. The absolute configurations of (+)-thalictrifoline and (+)-corydalic acid methyl ester. Total synthesis of (+)-thalictrifoline. Tetrahedron Lett. 1981, 22, 2333-2336. [CrossRef]

14. Cushman, M.; Chen, J.K. Utilization of the 1-ferrocenyl-2-methylpropyl substituent as a chiral auxiliary in the asymmetric syntheses of the benzophenanthridine alkaloids (+)- and (-)-corynoline. J. Org. Chem. 1987, 52, 1517-1521. [CrossRef]

15. Chunyang, Y.; Xiaolong, L.; Si, C.; Mingyao, L.; Weiqiang, L.; Xiyun, Y. Natural product corynoline suppresses melanoma cell growth through inducing oxidative stress. Phytother. Res. 2020, 10, 2766-2777. [CrossRef]

16. Burdzhiev, N.T.; Stanoeva, E.R. Reaction between glutaric anhydride and N-benzylidenebenzylamine, and further transformations to new substituted piperidin-2-ones. Tetrahedron 2006, 62, 8318-8326. [CrossRef]

17. Yu, N.; Deprez, B.; Gesquiere, J.C. Lewis acid-induced reaction of homophthalic anhydride with imines: A convenient synthesis of trans-isoquinolonic acids. Tetrahedron Lett. 1998, 39, 829-832. [CrossRef]

18. Vara, Y.; Bello, T.; Aldaba, E.; Arrieta, A.; Pizarro, J.L.; Arriortua, M.I.; Lopez, X.; Cossío, F.P. Trans-Stereoselectivity in the Reaction between Homophthalic Anhydride and Imines. Org. Lett. 2008, 10, 4759-4762. [CrossRef]

19. Ghorbani-Choghamarani, A.; Hajjami, M.; Norouzi, M.; Abbasityula, Y.; Eigner, V.; Dusek, M. Diastereoselective and one-pot synthesis of trans-isoquinolonic acids via three-component condensation of homophthalic anhydride, aldehydes, and ammonium acetate catalyzed by aspartic acid. Tetrahedron 2013, 69, 6541-6544. [CrossRef]

20. Howard, S.Y.; Di Maso, M.J.; Shimabukuro, K.; Burlow, N.P.; Tan, D.Q.; Fettinger, J.C.; Malig, T.C.; Hein, J.E.; Shaw, J.T. Mechanistic Investigation of Castagnoli-Cushman Multicomponent Reactions Leading to a Three-Component Synthesis of Dihydroisoquinolones. J. Org. Chem. 2021, 86, 11599-11607. [CrossRef]

21. Yadav, J.S.; Reddy, B.V.S.; Saritha Raj, K.; Prasad, A.R. Room temperature ionic liquids promoted three-component coupling reactions: A facile synthesis of cis-isoquinolonic acids. Tetrahedron 2003, 59, 1805-1809. [CrossRef]

22. Yu, N.; Poulain, R.; Gesquiere, J.C. A One-Pot Synthesis of Tetrahydroisoquinolonic Acids from Aldehydes and Amines in Trimethylorthoformate. Synlett 2000, 3, 355-356. [CrossRef]

23. Wang, L.; Liu, J.; Tian, H.; Qian, C.; Sun, J. One-Pot Synthesis of cis-Isoquinolonic Acid Derivatives via Three-Component Reaction of Homophthalic Anhydride with Aldehydes and Amines using Ytterbium(III) Triflate as Catalyst. Adv. Synth. Catal. 2005, 347, 689-694. [CrossRef]

24. Azizian, J.; Mohammadi, A.A.; Soleimani, E.; Karimi, A.R.; Mohammmadizahed, M.R. A stereoselective three-component reaction: One-pot synthesis of cis-isoquinolonic acids catalyzed by silica sulfuric acid under mild and heterogeneous conditions. J. Heterocycl. Chem. 2006, 43, 187-190. [CrossRef] 
25. Reza Karimi, A.; Pashazadeh, R. Sulfonic Acid Functionalized Silica: A Mild, Reusable and Efficient Heterogeneous Catalyst for the Highly Diastereoselective Synthesis of cis-Isoquinolonic Acids. Synthesis 2010, 3, 437-442. [CrossRef]

26. Azizian, J.; Mohammadi, A.A.; Karimi, A.R.; Mohammadizadeh, M.R. A Stereoselective Three-Component Reaction: $\mathrm{KAl}\left(\mathrm{SO}_{4}\right)_{2} \cdot 12 \mathrm{H}_{2} \mathrm{O}$, an Efficient and Reusable Catalyst for the One-Pot Synthesis of cis-Isoquinolonic Acid. J. Org. Chem. 2005, 70 , 350-352. [CrossRef]

27. Ali Reza, K.; Hamid Reza, M.; Rahim, P. L-Proline-catalyzed diastereoselective synthesis of cis-isoquinolonic acids and evaluation of their neuroprotective effects. Tetrahedron Lett. 2012, 53, 3440-3443. [CrossRef]

28. Colomer, I.; Chamberlain, A.E.R.; Haughey, M.B.; Donohoe, T.J. Hexafluoroisopropanol as a highly versatile solvent. Nat. Rev. Chem. 2017, 1, 0088. [CrossRef]

29. An, X.D.; Xiao, J. Fluorinated Alcohols: Magic Reaction Medium and Promoters for Organic Synthesis. Chem. Rec. 2020, 20, 142-161. [CrossRef]

30. Jarvis, C.L.; Hirschi, J.S.; Vetticatt, M.J.; Seidel, D. Catalytic enantioselective synthesis of lactams through formal [4+2] cycloaddition of imines with homophthalic anhydride. Angew. Chem. Int. Ed. 2017, 56, 2670-2674. [CrossRef]

31. Njiojob, C.N.; Rhinehart, J.L.; Bozell, J.J.; Long, B.K. Synthesis of Enantiomerically Pure Lignin Dimer Models for Catalytic Selectivity Studies. J. Org. Chem. 2015, 80, 1771-1780. [CrossRef]

32. Hazra, S.; Kushawaha, A.K.; Yadav, D.; Dolui, P.; Deb, M.; Elias, A.J. Table salt as a catalyst for the oxidation of aromatic alcohols and amines to acids and imines in aqueous medium: Effectively carrying out oxidation reactions in seawater. Green Chem. 2019, 21, 1929-1934. [CrossRef]

33. Dar'in, D.; Bakulina, O.; Chizhova, M.; Krasavin, M. New Heterocyclic Product Space for the Castagnoli-Cushman ThreeComponent Reaction. Org. Lett. 2015, 17, 3930-3933. [CrossRef] [PubMed]

34. Borrione, E.; Prato, M.; Scorrano, G.; Stivanello, M. Synthesis and cycloaddition reactions of ethyl glyoxylate imines. Synthesis of substituted furo-[3,2-c]quinolines and $7 \mathrm{H}$-indeno[2,1-c]quinolines. J. Heterocycl. Chem. 1988, 25, 1831-1835. [CrossRef] 